\title{
THE SHORT MESSAGE MANAGEMENT SYSTEM BASED ON J2ME
}

\author{
Shumin Zhou ${ }^{*}$, Guoyun Zhong, Tiantai Zhang \\ School of Information Engineering, East China Institute of Technology, Fuzhou, China, \\ 344000 \\ "Corresponding author, Address: School of Information Engineering, East China Institute of \\ Technology, 56 Xufu Road, Fuzhou, Jiangxi Province, China, 344000, Tel: +86-794- \\ 8258390, Fax:+86-794-8258390, Email: smzhou@ecit.edu.cn
}

Abstract: In order to apply Java to mobile communication device and embedded device, the J2ME is issued by SUN. It is also be used on $3 \mathrm{G}$ mobile multiplication service by telecommunication company. The paper introduces the methods and processes of developing short message management system adopt J2ME and Siemens Mobility Toolkit 6688i. The system applies 12 classes to realize message grouping, message list and message editing. By this system, the efficiency of the short message management in the mobile phone is increased.

Keywords: J2ME, Short Message, MIDlet

\section{INTRODUCTION}

Short Message Service (SMS) (ETSI/TC, 1997) has become a mature wireless communication service. SMS provides a connectionless transfer of messages with low-capacity and low-time performance (Gerd et al., 2003). In order to improve the application of mobile phone, the related programming languages were issued. The development of the mobile phone software has mainly experienced three stages. At first stage, it just focused on the basic voice service, afterwards the simple value-added service applied, then the function which access Internet by the WAP was developed. J2ME is issued by SUN in order to apply Java to mobile communication device and embedded device and consumed electrical equipment (Microsystems, 1999). Two problems can be solved by J2ME: (1) Java language runs across different platform, software development businessman develops related 
application easily, so it can also be installed conveniently to mobile phone; (2) J2ME has offered high-grade programming of the internet protocol such as HTTP, TCP and UDP, so JAVA application can be accessed on Internet freely. In the mobile communication toady, global operators are arranging 3G strategy, they have met the problem how to consume these bandwidth increased. Now J2ME is seemed as a best solving scheme, offer the multiplication service of mobile data business based on J2ME for user. Mobile multiplication service, such as mobile business, mobile information service, mobile internet business and Virtual special net business, mobile office, mobile medical treatment, will become true by using J2ME.

Short message is now mobile service used the most broadly exclude the conversation of speech sound outside, therefore this paper adopts J2ME to realize Short message management system, makes the JAVA user of mobile telephone carry out various managements for short message.

\section{THE STRUCTURE OF CLDC/MIDP}

J2ME is composed of two major components: configuration and profile, which together establish an implementation specification for consumer electronics and embedded device manufactures (Sun Microsystems, 2002). J2ME is built up on a three-layered architecture (from bottom to top): JVM Layer, Configuration Layer, and Profile Layer. The JVM layer is an implementation of a specific JVM that is customized for an operating system on small devices. For example, the K virtual machine (KVM) is a highly portable virtual machine, designed for small memory, limited-resource, and network-connected devices such as cellular telephones, pagers, and small point of sale (POS) systems. The configuration layer defines a minimum set of Java virtual machine features and Java class libraries available on a particular category of devices. At present there are two J2ME configurations: the Connected Limited Device Configuration (CLDC) and the Connected Device Configuration (CDC). CLDC is designed for developing applications on resource-constrained devices with parsimonious memory, say, less than $512 \mathrm{~KB}$. On the contrary, CDC is developed for embedded devices with relatively larger amounts of memory and more robust resources like set-top box, screen phone or refrigerator. The configuration layer also specifies core class libraries of device-dependence so that the applications can be developed regardless of the devices they will be deployed to.

The top layer is the so-called profile layer, which is independent on the connection layer. A profile is an industry-defined specification of the Java APIs which fits for a specific industry or class of devices. For example, the Mobile Information Device Profile (MIDP) coupled with CLDC specifies the issues of user interface, persistent storage, and networking for mobile 
information devices such as cellular phones. The so-called K-Java is a complete J2ME application run-time environment fully adhering to the CLDC specification whereas PersonalJava (the so-called pJava) is partly compatible to the CDC specification since the review of CDC specification is still underway. The former one is suitable for consumer electronics with colorful terminals like Windows CE devices while the latter is good for resource-constrained devices such as PalmPilot. J2ME is designed on the basis of philosophy that applications developed on a particular profile can be deployed straightforward onto different configurations without having to modify the code. However, such ideal has not been realized currently. In this paper, we adopt the MIDP/CLDC approach to develop the surveillance system on the K-Java environment. It is expected the hurdle of platform heterogeneity can be removed when CDC is finalized.

Table layer also contain a group of API, defined aiming at mainly the specific equipment of certain a clan department. Table layer realizes on specific disposition layer, programmer takes the responsibility for compiling application program on specific Table layer. J2ME has defined many Tables, but only MIDP has realization reference, MIDP (Mobile Information Device Pro2file) is designed for mobile telephone and two-way paging equipment. It is very similar that MIDP application is called as MIDlet with Java Applet, they have similar life cycle. The life cycle of MIDlet can 3 stages: (1) start, get resource and run; (2) suspend, release resource and change to wait state; (3) die out, release the resource owned, finish thread and all activities. MIDP and CLDC have formed a complete configuration together. Fig. 1 has shown the structure of CLDC/MIDP of development of mobile telephone program.

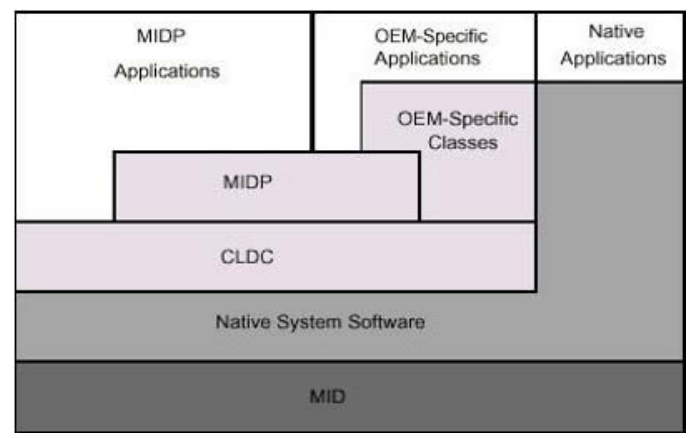

Figure 1. The structure of CLDC/MID 
3. THE DEVELOPMENT OF SHORT MESSAGE MANAGEMENT SYSTEM

Developing a MIDlet program or other wireless Java application software following three steps: (1) compile application software; (2) test application software in simulator; (3) download the compiled software to the mobile phone, then operate and test.

Short message management system belongs to MIDlet (Ganhua et al., 2003), which is a short message management system developed with API of Siemens. Purpose of compiling the system is that user can manage the short message on mobile telephone effectively, its major functions include dividing short messages into groups, the editor, insert, delete operation of short message list and message content etc., additionally realize short message to dispatch operation.

\subsection{Development tool and development environment}

The popular J2ME development tools include: (1) SUN Wireless Toolkit + UltraEdit + various simulators; (2) Builder + Mobile Set + Nokia SDK; (3) JCreator + MotoSDK. The JDK + UltraEdit + SMTK6688i was used to develop J2ME which is provide by Simens in our system.

\subsection{System profile}

The system is composed of 12 operating class, can realize management of short message and some related operations based on this function. User can use the groups that the system has to establish related short message list, also can delete the system groups, establish short message groups, and also can change its name.

According to each groups in short message list, you can delete and add and modify. They are the operations according to mobile telephone of the user and short message contents. After looking over the content of short message, user can also edit, delete and modify, can sent this short message to the current user of the mobile telephone. Because it realizes by a class which improves function based on com.siemens.mp.io. of Siemens class File, we must converse code before we do file operation, that is, we must change ASCII yard to UTF28 or Unicode coding before stocking.

Above analyzed, we can get the structure of systematic function as Fig. 2 shows. 


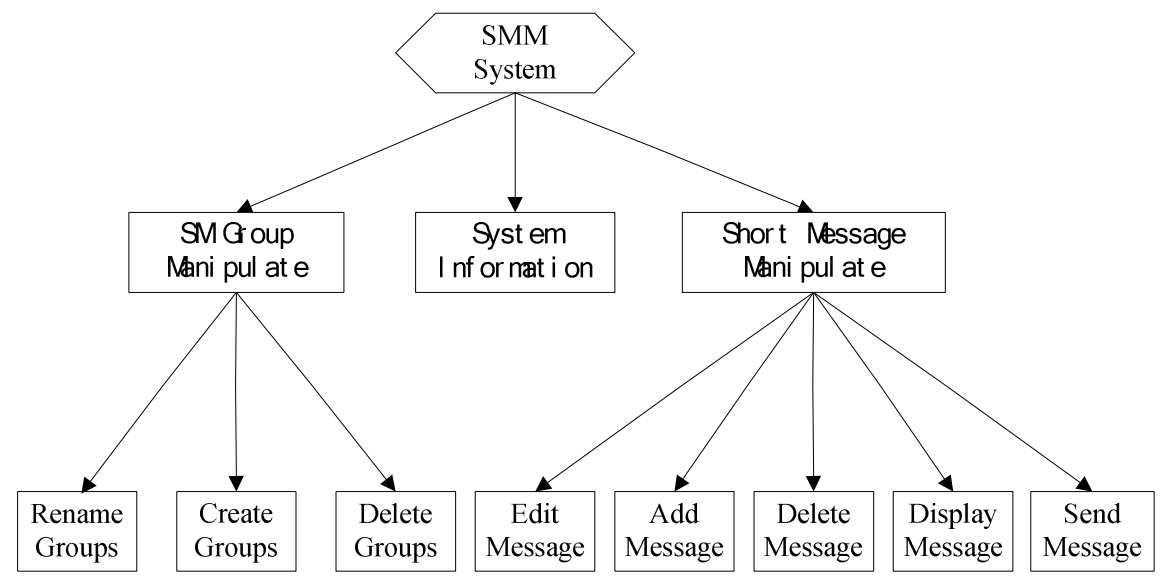

Figure 2. SMM system structure

\subsection{The file operation of short message group}

Because the J2ME language of standard do not offer file operating class, this system used a ManipFile class that compiled on com.siemens.mp.io.File of Siemens to realize related file operation. SMM.java class is the frame of whole system, its major function is use SMM structure function to example the example of current program, its value is this indicator of current program, this value will be used for getting to show equipment and system the sentence during the whole process. In addition, the SMM.java class realizes the interface between the systemc and application program management equipment, application program management equipment is the software that installed on MIDP equipment in advance and acts the role of operation environment. It can use the life period method of MIDlet in order to control the state of MIDlet. SMMInfo.java class shows copyright of the system, the informations and helps by inheriting Form. Add two buttons on Form, it will respond to the operation that user press one button by realizing CommandListener interface, for example, it will enter to show short message list after pressing cmdEnter, and exit cmdExit.

ManipFile. java class is the key class concerned with file operating during the system operation course, during the system operation course, all operations concerned with file operating have corresponding definitions in this class. File the structure function of operating class, open fileName file, if file does not exist, build a new fileName file $\mathrm{f}$ isNew is true, otherwise, it throw unusual and quit program. After opening file, structure function with initialize operation such as the descriptions of the file, the length and the name of this file, and the current file indicator etc. According to the file indicator location that offers, read the content as (iStart2iEnd) from file and return this string. In current file, read one row of character from nStart, and 
return this string. Write in the opened iPos of the file After change the string str to byte type. Write the string in a new file fileName in. In this method, must establish fileName (if this file existence unusual, throw it and quit), after change str to byte type, write in the file, close this file finally.

\subsection{Storage of short message}

The operations of message groups includes groups show, change group's name, delete group and build new group. Group show is realized by SMGroupList.java class. The course of message groups shows is that open Index.txt of stock short message, read their names, use GetGroupList ( ) method read a row every time and show.

Short message groups'names modification is sent the selected message group's name to the groups' name modification class as parameter. After modification, must write the result of modification to stock groups Index. txt file.During the course of stocking, must use the method InsertStrToFile of ManipFile. java class. Adding short message groups is also establishment the method of groups requirement completion after need should groups name join. txt file end, use ManipFile. FileAppend of java class ( ) method.

In the course of deleting message groups, must conside the possibility of short message groups, because before deleting, should warn; If confirme to deleted, besides delete the current groups' names from Index. txt file, and delete the file stocking current message. Because short message groups file named with sequence number, so just according to current groups sequence number, we can find very easily short message groups file and delete it, then, rename short message files after this file again according to digital order.

If some errors such as transfer failure, invalidate retry occurred in SM sending module, SM receipting module and link maintain module, it will be terminated in the corresponding module, The manage module will take some measures such as resume the link, restart the module and so on according to the terminate sate.

\subsection{The list of short message}

Message list management have realized the operations - show, delete, modification, add etc in divided group messages. Short message list show by SMSList.java class. Construct function shows current message list and open corresponding file to read short message list of this groups and show in Form by recepting the parameter and sequence number of the title. In the course of adding short message content, we must input short message content and mobile telephone which is made up with 11 numbers, which is controlled by the sentence "numTxtF ld = newTextField ("the number of mobile telephone" “,", 11, TextField. PHONENUMBER)". Because generally 
length of the message content is also limited, which can be control according to specific condition by the sentence "smsTxtFld = new TextField ("short letter content" “,", 200, TextField. ANY) (Maximum will be 200 characters short the length definition of news content)". The editor of short message list is similar with short message groups. During the deleting of short message list, we must consider the length of short message and find out the location of this message, then carry out related insert operation, replace the short message content to delete with space. List show and content input interface as Fig. 3 show.
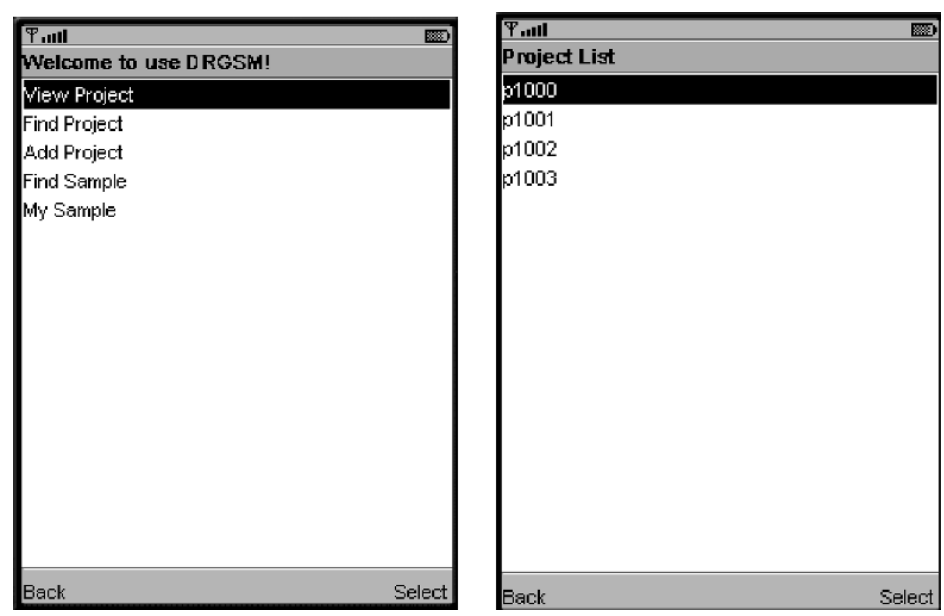

Figure 3. Short Message List

\section{CONCLUSIONS AND FUTURE WORKS}

This system is used Siemens API for special purpose to develop, in the process of development, it uses Siemens6688i simulator to carry out debug and operate, and test successfully on the mobile telephone of Siemens, if it to be used in other simulator or the mobile telephone excepted Siemens, need to carry out further modification and test.

\section{REFERENCES}

ETSI/TC, "Use of Data Terminal Equipment-Data Circuit Terminating; Equipment (DTEDCE) Interface for Short Message Service (SMS) and Cell Broadcast Service (CBS)," v. 5.3.0, Tech. rep., Rec. GSM 07.05, 1997. 
Gerd Beuster, Thomas Kleemann and Bernd Thomas, "Multi-Agent Location Based Information Systems for Mobile Users in 3G Networks" Submitted to WI2003-6, International Tagung Wirts chafts informatik 2003.

Ganhua Li and Yunhui Liu, Robotics. A distributed and adaptive data flow system for SMS[C]. Intelligent Systems and Signal Processing, vol. 2 1350, 2003

Sun Microsystems, J2ME CLDCAPI 1.1, Inc. 2003

Mobile Information Device Profile (MIDP). http://Java.sun.com/products/midp/, 2002-09. [EB/OL]

J. Desbonnet, P.M. Corcoran, System architecture and implementation of a CE Bus/Internet gateway, IEEE Transactions on Consumer Electronics 43 (1997) 1057-1062.

J. Ellis, J2ME Web Services Specification, Sun Microsystems, Inc., 4150 Network Circle Santa Clara, CA 95054, 2003 July, http://www.sum.com.

Y.-R. Haung, Y.-B. Lin, J.-M. Ho. Performance analysis for voice/data integration on a finite buffer mobile system, IEEE Trans. Veh. Technol. 49 (2) (2000) 367-378. 